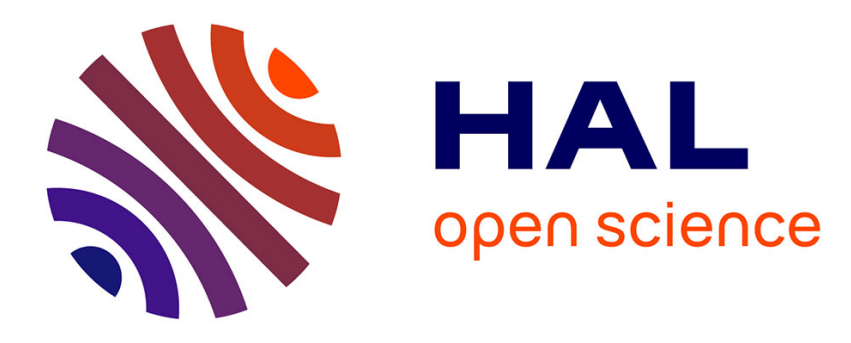

\title{
Graph of hippocampal subfields grading for Alzheimer's disease prediction
}

Kilian Hett, Vinh-Thong Ta, José V Manjón, Pierrick Coupé

\section{To cite this version:}

Kilian Hett, Vinh-Thong Ta, José V Manjón, Pierrick Coupé. Graph of hippocampal subfields grading for Alzheimer's disease prediction. Machine Learning in Medical Imaging (MICCAI), Sep 2018, Granada, Spain. hal-01859257

\section{HAL Id: hal-01859257 \\ https://hal.science/hal-01859257}

Submitted on 21 Aug 2018

HAL is a multi-disciplinary open access archive for the deposit and dissemination of scientific research documents, whether they are published or not. The documents may come from teaching and research institutions in France or abroad, or from public or private research centers.
L'archive ouverte pluridisciplinaire HAL, est destinée au dépôt et à la diffusion de documents scientifiques de niveau recherche, publiés ou non, émanant des établissements d'enseignement et de recherche français ou étrangers, des laboratoires publics ou privés. 


\title{
Graph of hippocampal subfields grading for Alzheimer's disease prediction
}

\author{
Kilian Hett ${ }^{1,2}$, Vinh-Thong Ta ${ }^{1,2,3}$, José V. Manjón ${ }^{4}$, Pierrick Coupé ${ }^{1,2}$, and \\ the Alzheimer's Disease Neuroimaging Initiative* \\ 1 Univ. Bordeaux, LaBRI, UMR 5800, PICTURA, F-33400 Talence, France \\ 2 CNRS, LaBRI, UMR 5800, PICTURA, F-33400 Talence, France \\ ${ }^{3}$ Bordeaux INP, LaBRI, UMR 5800, PICTURA, F-33600 Pessac, France \\ 4 Universitat Politècnia de València, ITACA, 46022 Valencia, Spain
}

\begin{abstract}
Numerous methods have been proposed to capture early hippocampus alterations caused by Alzheimer's disease. Among them, patch-based grading approach showed its capability to capture subtle structural alterations. This framework applied on hippocampus obtains state-of-the-art results for AD detection but is limited for its prediction compared to the same approaches based on whole-brain analysis. We assume that this limitation could come from the fact that hippocampus is a complex structure divided into different subfields. Indeed, it has been shown that $\mathrm{AD}$ does not equally impact hippocampal subfields. In this work, we propose a graph-based representation of the hippocampal subfields alterations based on patch-based grading feature. The strength of this approach comes from better modeling of the inter-related alterations through the different hippocampal subfields. Thus, we show that our novel method obtains similar results than state-of-the-art approaches based on whole-brain analysis with improving by 4 percent points of accuracy patch-based grading methods based on hippocampus.
\end{abstract}

Keywords: Hippocampal subfields, Patch-based grading, Graph-based method, Alzheimer's disease classification, Mild Cognitive Impairment

\section{Introduction}

Alzheimer's disease (AD) is the most prevalent dementia for the older adults. This disease leads to an irreversible form of neurodegenerative process causing mental dysfunctions. Neuroimaging studies performed on AD patients has revealed that $\mathrm{AD}$ causes structural brain changes. These changes are characterized by an accelerated neural and synapse losses, which are advanced when the diagnosis is established. A presymptomatic phase of AD is named mild cognitive

\footnotetext{
^ Data used in preparation of this article were obtained from the Alzheimer's Disease Neuroimaging Initiative (ADNI) database (adni.loni.usc.edu). As such, the investigators within the ADNI contributed to the design and implementation of ADNI and/or provided data but did not participate in analysis or writing of this report. A complete listing of ADNI investigators can be found at: http://adni.loni.usc.edu/wp-content/uploads/how_to_apply/ADNI_Acknowledgement_List.pdf.
} 
impairment (MCI). Patients having MCI suffer from amnesia and light mental issues that do not impact their daily life. Moreover, MCI patients can return to a cognitive normal status or progress to dementia. Predicting this conversion is a challenging task but can make easier the design of clinical trials and accelerate the development of new therapies.

It has long been known that in-vivo imaging with magnetic resonance imaging (MRI) can detect structural alterations such as atrophy of grey matter volume. Thus, several methods were proposed. On the one hand, voxel-based morphometry (VBM) methods were designed to detect the most discriminant regions at a voxel scale [18]. On the other hand, region-based methods were proposed to analyze specific brain structures. These analysis are usually conducted with volume, shape or thickness measurements [18]. It is interesting to note that both of VBM and region-based approaches have demonstrated that the medial temporal lobe especially the hippocampus is the area having the earliest alterations. However, although the hippocampus volume is one of the criterion that can be used to confirm the diagnosis of $\mathrm{AD}$ in clinical routines, it does not provide acceptable performance for the prediction of $\mathrm{AD}$ conversion. Recently, more advanced methods were proposed to detect subtler structural modifications of the hippocampus [11. Among them, patch-based grading (PBG) framework was proposed to better capture subtle brain alterations caused by $\mathrm{AD}$ [2]. The main idea of PBG methods is to detect modifications in a cubic fixed-size area named patch. PBG methods applied to the hippocampus have demonstrated state-of-the-art performances for $\mathrm{AD}$ detection and prediction compared to others methods based on the hippocampus [2]14/5].

However, the hippocampus is not a heterogeneous structure. Indeed, the hippocampus is composed of different subfields having distinct characteristics. A common definition of hippocampal subfields [17] divided them into the subiculum, the cornu ammonis (CA), and the dentrate gyrus (DG). The CA represents the most prominent area. It is usually divided into four regions: CA1, CA2, CA3, and CA4. The CA1 is also composed of several layers, such as the stratum pyramidale (SP), stratum radiatum (SR), stratum molecular (SM), and stratum lacunosum (SL). Moreover, postmortem and animal-based studies showed that hippocampal subfields are not impacted equally. Those studies showed that CA1 and subiculum are the most impacted at the last stages of AD [6]15. Consequently, we assume that a straightforward whole hippocampus analysis could limit the prediction performances. An analysis of the hippocampus at a finner scale such as hippocampal subfields may improve prediction.

To confirm this assumption, we propose a novel method to better capture AD signature within hippocampal subfields. Our method integrates patch-based grading features into a new graph-based model that provides efficient representation of the inter-related alterations through the hippocampus. We demonstrate that our novel approach improves patch-based grading method applied into the hippocampus by 4 percent points of accuracy and obtains state-of-the-art results compared to last advanced methods based on whole brain analysis for AD prediction. 


\section{Materials and Methods}

Dataset Data used in this work were obtained from Alzheimer's Disease Neuroimaging Initiative (ADNI) dataset ${ }^{1}$ ADNI is a North American campaign launched in 2003 with aims to provide MRI, positron emission tomography scans, clinical neurological measures and other biomarkers. The data used in this study are all the baseline T1-weighted (T1-w) MRI of the ADNI1 phase. This dataset includes AD patients, MCI and cognitive normal (CN) subjects. The group of MCI is composed of subjects who have abnormal memory dysfunctions and embed two groups; the first one is composed with patients having stable MCI (sMCI) and the second one with patients having progressive MCI (pMCI) who converted in the following 36 months after the baseline. The information of the dataset used in our work is summarized in Table 1.

Table 1. Description of the dataset used in this work. Data are provided by ADNI.

\begin{tabular}{lcccc}
\hline Characteristic / Group & CN & sMCI & pMCI & AD \\
\hline Number of subjects & 213 & 90 & 126 & 130 \\
Ages (years) & $75.7 \pm 5.0$ & $74.9 \pm 7.5$ & $73.7 \pm 7.0$ & $74.1 \pm 7.7$ \\
Sex (M/F) & $108 / 105$ & $58 / 32$ & $68 / 58$ & $64 / 66$ \\
MMSE & $29.1 \pm 1.0$ & $27.6 \pm 1.7$ & $26.5 \pm 1.6$ & $23.5 \pm 1.9$ \\
\hline
\end{tabular}

Preprocessing First, each image was preprocessed with a method based on an advanced pipeline providing: (a) a denoising step with an adaptive non-local mean filter [8], (b) an affine registration in the MNI space [1], (c) a correction of the image inhomogeneities [16] and (d) an intensity normalization. Second, segmentation of hippocampal subfields was performed with HIPS. This method is based on a combination of non-linear registration and patch-based label fusion [10]. This method uses a training library based on a dataset composed of high resolution T1w images manually labeled according to the protocol proposed by [17]. Consequently, to perform the segmentation, the ADNI images are upsampled with a local adaptive super resolution method to fit in the training image resolution 3. The method provides automatic segmentation of hippocampal subfields gathered into 5 regions: Subiculum, CA1SP, CA1SR-L-M, CA2-3 and CA4/DG. Finally, visual quality control was conducted to remove all wrong segmentations from the dataset. Moreover, to prevent any bias in the dataset, the quality control was performed without the pathological status of each subject.

Computation of patch-based grading biomarkers To capture anatomical similarities of alterations caused by $\mathrm{AD}$, we use the patch-based grading framework [2]. PBG framework provides at each voxel a score between -1 and 1 related

\footnotetext{
1 http://adni.loni.ucla.edu
} 


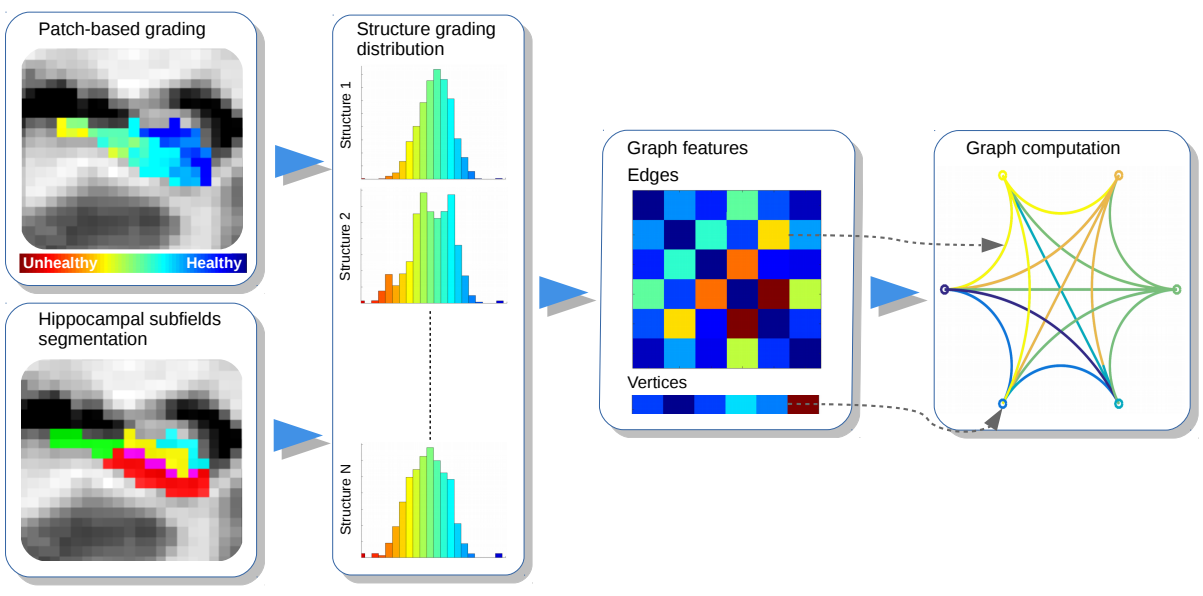

Fig. 1. Illustration of the graph construction. From left to right, for each hippocampal region an estimation of the density probability of PBG values is computed. Next, histograms are used to built our graph of hippocampal subfields grading. Graph edges represent the distances between structure grading distribution while vertices represents the mean grading value for a given hippocampal regions (i.e., hippocampus, subiculum, CA1-SP, etc.)

to the alteration severity. The patch-based grading value $g$ at $x_{i}$ is defined as:

$$
g_{x_{i}}=\frac{\sum_{t_{j} \in K_{i}} w\left(P_{x_{i}}, P_{t_{j}}\right) p_{t}}{\sum_{t_{j} \in K_{i}} w\left(P_{x_{i}}, P_{t_{j}}\right)}
$$

where $P_{x_{i}}$ and $P_{t_{j}}$ represent the cubic patches surrounding the voxel $i$ of the test subject image $x$ and the voxel $j$ of the template image $t$, respectively. The template $t$ comes from a training library composed of $\mathrm{CN}$ subjects and $\mathrm{AD}$ patients. $p_{t}$ is the pathological status set to -1 for patches extracted from $\mathrm{AD}$ patients and to 1 for those extracted from $\mathrm{CN}$ subjects. $K_{i}$ is a set of the most similar $P_{t_{j}}$ patches to $P_{x_{i}}$ found in the training library. The anatomical similarity between the test subject $x$ and the training library is estimated by a weight function $w\left(P_{x_{i}}, P_{t_{j}}\right)=\exp \left(-\left\|P_{x_{i}}-P_{t_{j}}\right\|_{2}^{2} /\left(h^{2}+\epsilon\right)\right)$, where $h^{2}=\min _{t_{j}} \| P_{x_{i}}-$ $P_{t_{j}} \|_{2}^{2}$ and $\epsilon \rightarrow 0$.

Graph construction First, the grading process is carried out over the whole hippocampus. Afterwards, the corresponding hippocampal subfield segmentations is used to fuse grading values and to built our graph (see Fig. 1). An undirected graph is defined as $G=(V, E, \Gamma, \omega)$, where $V=\left\{v_{1}, \ldots, v_{N}\right\}$ is the set of vertices for the $N$ considered hippocampal regions (i.e., the whole hippocampus and each on its hippocampal subfields) and $E=V \times V$ is the set of edges. Thus, in our model the vertices represents the mean of the grading values for a given hippocampus region while the edges are based on grading distribution distances between two hippocampal regions. 
The probability distributions of PBG values are estimated with a histogram $H_{v}$ for each hippocampus region $v$. The number of bins is set following the Sturge's rule. For each vertex we assign a function $\Gamma: V \rightarrow \mathbb{R}$ defined as $\Gamma(v)=$ $\mu_{H_{v}}$, where $\mu_{H_{v}}$ is the mean of $H_{v}$. For each edge we assign a weight given by the function $\omega: E \rightarrow \mathbb{R}$ defined as $\omega\left(v_{i}, v_{j}\right)=\exp \left(-d\left(H_{v_{i}}, H_{v_{j}}\right)^{2} / \sigma^{2}\right)$ where $d$ is the Wasserstein distance with $L_{1}$ norm that showed best performance during our experiments.

In this work, we used the Elastic Net regression (EN) method that provides a sparse representation of the most discriminative edges and vertices, and thus enables to reduce the feature dimensionality by capturing the key regions and the key relationships between the different hippocampus regions (see Fig. 1). Finally, after z-score normalization, a concatenation of the two feature vectors is given as input of EN feature selection method.

Details of implementation The most similar patches were extracted with a patch-match method [4]. We used the parameters proposed in [5] for the sizes of the patches and $K_{i}$. This results in a hippocampus grading computation in about 1 second. Next, the age effect is corrected using linear regression estimated on CN population. The EN method is computed with the SLEP package [7. The classifications were obtained with the random forest method (RF) ${ }^{5}$, All features were normalized using z-score before selection and classification methods. In our experiments, we performed sMCI versus pMCI classification. The EN features selection and the classifier were trained with $\mathrm{CN}$ and $\mathrm{AD}$. Indeed, as shown in [14], using $\mathrm{CN}$ and $\mathrm{AD}$ to train the feature selection method and the classifier enables to better discriminate sMCI and pMCI subjects. Furthermore, it also enables to limit bias and over-fitting problem without cross-validation step. However, 100 runs were performed to decrease the inner variability of RF. Mean area under curve (AUC), accuracy (ACC), balanced accuracy (BACC), sensibility (SEN), and specificity (SPE) are provided as results in Tables 2 and 3.

\section{Results and Discussions}

First, a comparison of the prediction performances was conducted with PBG applied into the whole hippocampus as proposed in [2], the hippocampal subfields with EN selection, and our proposed method (see Table 2). The average of PBG values into each region (i.e., whole hippocampus and hippocampal subfields) were used. For hippocampal subfields features, the most relevant subfields selected are the subiculum, and the two definitions of CA1 (i.e., CA1-SP and CA1-SRLM, see Figure 2p. It is very interesting that hippocampal subfields selected by EN method are in line with previous studies which have shown CA1 and subiculum are the subfields having the most significant atrophy in late stages of $\mathrm{AD}[6] 15]$. PBG based on the whole hippocampus structure obtains $76.8 \%$ of

\footnotetext{
5 http://code.google.com/p/randomforest-matlab
} 


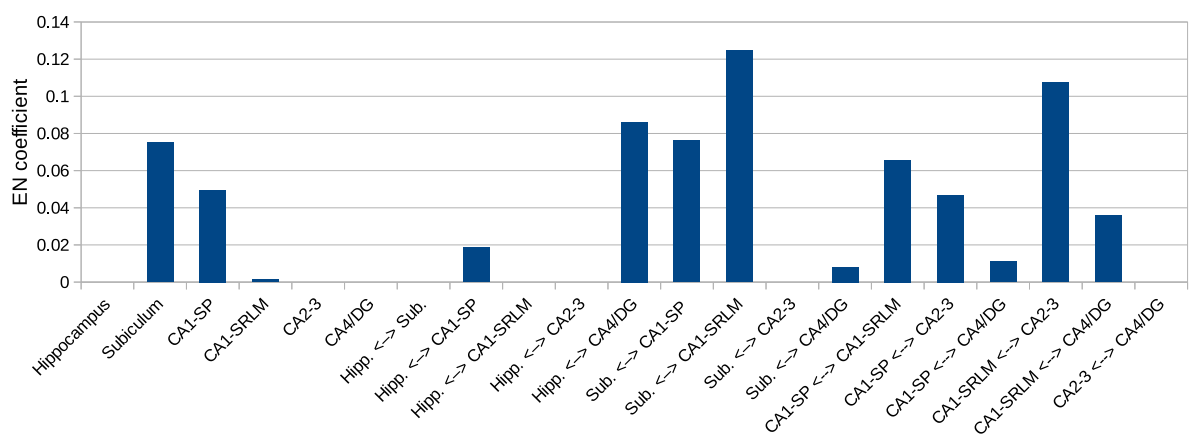

Fig. 2. Graphic representing the coefficients estimated by EN method from the vector of features computed by our graph-based method after z-score normalization. The first six labels represent hippocampal regions and the others represents relationships of these regions. It is interesting to note that selected structures are in line with previous hippocampal subfields investigations.

Table 2. Comparison of different hippocampal PBG approaches is presented. First, PBG applied within the whole hippocampus is provided as the baseline. Second, the best hippocampal subfield features selected by EN method. Finally, results provided by our graph-based of hippocampal subfields grading. This comparison shows that our proposed method improves AUC, ACC, BACC, and SEN compared to other approaches. All results are given in percentage.

\begin{tabular}{lccccc}
\hline Methods & AUC & ACC & BACC & SEN & SPE \\
\hline Hippocampus & $76.8 \pm 0.2$ & $70.3 \pm 0.0$ & $70.6 \pm 0.0$ & $69.0 \pm 0.0$ & $72.2 \pm 0.0$ \\
Hipp. subfields EN & $77.1 \pm 0.2$ & $71.1 \pm 0.4$ & $71.4 \pm 0.4$ & $69.5 \pm 0.6$ & $\mathbf{7 3 . 2} \pm \mathbf{0 . 5}$ \\
Proposed method & $\mathbf{7 8 . 2} \pm \mathbf{0 . 2}$ & $\mathbf{7 4 . 7} \pm \mathbf{0 . 4}$ & $\mathbf{7 4 . 3} \pm \mathbf{0 . 5}$ & $\mathbf{7 7 . 1} \pm \mathbf{0 . 5}$ & $71.4 \pm 0.9$ \\
\hline
\end{tabular}

AUC, $70.3 \%$ of ACC and is more specific than sensitive. The averages of PBG values within subiculum, CA1-SP, and CA1-SRLM obtain $77.1 \%$ of AUC, $71.1 \%$ of ACC and improve specificity compare to the hippocampus. Thus, the concatenation of mean grading values based on each hippocampal subfields selected with a EN method slightly increases the prediction performances of AD. However, our proposed graph-based method improves by 1.4 percent points with AUC and 4.4 percent points of ACC compared to the hippocampus. Our graph-based method also improves by 1.1 percent points with AUC and 3.6 percent points with ACC compared to the use of the most discriminant hippocampal subfields. Moreover, in both cases, our proposed method increases the sensibility of $\mathrm{AD}$ conversion.

Second, a comparison of our novel graph-based method based on hippocampal subfields and state-of-the-art methods based on the hippocampus, using similar ADNI1 dataset, is provided in the upper part of Table 3 . In this comparison, we included the original PBG method [2, a method based on multiple instance learning [13, and an advanced PBG method based on a sparse representation [14. The results demonstrate that our novel graph-based method obtains better results than all compared methods applied to the hippocampus. Indeed, to the 
Table 3. Comparison with state-of-the-art methods based on the hippocampus region and approaches based on a whole brain analysis using similar ADNI1 dataset and the same definition of sMCI/pMCI. These results show that our proposed method obtains best results compared to methods applied within the hippocampus. Moreover, compared to approaches based on a whole brain analysis, our method obtains competitive results. All results are given in percentage.

\begin{tabular}{lccccc}
\hline Methods & Registration & AUC & ACC & SEN & SPE \\
\hline Hippocampus & & & & & \\
\hline Original grading [2] & Affine & - & 71.0 & 70.0 & 71.0 \\
Multiple instance grading [13] & Affine & - & 70.4 & 66.5 & $\underline{73.1}$ \\
Sparse-based grading [14] & Non Linear & - & 69.0 & - & - \\
Proposed method & Affine & $\underline{\mathbf{7 8 . 2}}$ & $\underline{74.7}$ & $\underline{77.1}$ & 71.4 \\
\hline Whole brain & & & & & \\
\hline Voxel-based [9] & Non Linear & 76.6 & 74.7 & $\mathbf{8 8 . 8}$ & 51.6 \\
Sparse-based grading [14] & Non Linear & - & $\mathbf{7 5 . 0}$ & - & - \\
Deep ensemble learning [12] & Non Linear & 75.4 & 74.8 & 70.9 & $\mathbf{7 8 . 8}$ \\
\hline
\end{tabular}

best of our knowledge, state-of-the-art methods applied on hippocampus have obtained $71 \%$ of ACC for sMCI versus pMCI classification while our graph-based of hippocampal subfields grading obtains $74.7 \%$ of ACC.

Finally, in the lower part of Table 3 a comparison with state-of-the-art methods applied on the whole brain is provided. Our method is compared with a VBM approach [9], the advanced PBG method based on a sparse representation 14] and a recent deep ensemble learning method [12. This comparison shows that our novel graph of hippocampal subfields grading obtains comparable ACC and AUC than the last advanced approaches.

\section{Conclusions}

In this work, we proposed a new approach to better capture AD signature into the hippocampus. Our method is based on a graph-based representation of inter-related hippocampal subfields alterations. Alterations were captured with a patch-based grading framework while the relationships of alterations between the different subfields are based on histogram distances. We demonstrate that our method improves patch-based grading method based on hippocampus by 4 percent points for sMCI versus pMCI classification. Moreover, our novel approach obtains competitive results compared to state-of-the-art methods based on a whole brain analysis.

Acknowledgement This study has been carried out with financial support from the French State, managed by the French National Research Agency (ANR) within the project DeepVolbrain and in the frame of the investments for the future program IdEx Bordeaux (HL-MRI ANR-10-IDEX-03-02), Cluster of excellence CPU, TRAIL (BigDataBrain ANR-10-LABX- 57) and the Spanish DPI2017- 
Hett et al.

87743-R grant from the Ministerio de Economia, Industria y Competitividad of Spain.

\section{References}

1. Avants et al. A reproducible evaluation of ANTs similarity metric performance in brain image registration. NeuroImage, 54(3):2033-2044, 2011.

2. Coupé et al. Scoring by nonlocal image patch estimator for early detection of Alzheimer's disease. NeuroImage: Clinical, 1(1):141-152, 2012.

3. Coupé et al. Collaborative patch-based super-resolution for diffusion-weighted images. NeuroImage, 83:245-261, 2013.

4. Giraud et al. An optimized patchmatch for multi-scale and multi-feature label fusion. NeuroImage, 124:770-782, 2016.

5. Hett et al. Adaptive fusion of texture-based grading: Application to alzheimer's disease detection. In International Workshop on Patch-based Techniques in Medical Imaging, pages 82-89. Springer, 2017.

6. Kerchner et al. Hippocampal CA1 apical neuropil atrophy and memory performance in Alzheimer's disease. NeuroImage, 63(1):194-202, 2012.

7. Liu et al. Slep: Sparse learning with efficient projections. Arizona State University, 6(491):7, 2009.

8. Manjón et al. Adaptive non-local means denoising of MR images with spatially varying noise levels. Journal of Magnetic Resonance Imaging, 31(1):192-203, 2010.

9. Moradi et al. Machine learning framework for early MRI-based Alzheimer's conversion prediction in MCI subjects. NeuroImage, 104:398-412, 2015.

10. Romero et al. Hips: A new hippocampus subfield segmentation method. NeuroImage, 163:286-295, 2017.

11. Sørensen et al. Differential diagnosis of mild cognitive impairment and Alzheimer's disease using structural MRI cortical thickness, hippocampal shape, hippocampal texture, and volumetry. NeuroImage: Clinical, 13:470-482, 2017.

12. Suk et al. Deep ensemble learning of sparse regression models for brain disease diagnosis. Medical Image Analysis, 37:101-113, 2017.

13. Tong et al. Multiple instance learning for classification of dementia in brain MRI. Medical Image Analysis, 18(5):808-818, 2014.

14. Tong et al. A novel grading biomarker for the prediction of conversion from mild cognitive impairment to Alzheimer's disease. IEEE Transactions on Biomedical Engineering, 64(1):155-165, 2017.

15. Trujillo-Estrada et al. Early neuronal loss and axonal/presynaptic damage is associated with accelerated amyloid- $\beta$ accumulation in a $\beta \mathrm{pp} / \mathrm{ps} 1$ Alzheimer's disease mice subiculum. Journal of Alzheimer's Disease, 42(2):521-541, 2014.

16. Tustison et al. N4ITK: improved N3 bias correction. IEEE Transactions on Medical Imaging, 29(6):1310-1320, 2010.

17. Winterburn et al. A novel in vivo atlas of human hippocampal subfields using high-resolution 3T magnetic resonance imaging. NeuroImage, 74:254-265, 2013.

18. Wolz et al. Multi-method analysis of MRI images in early diagnostics of Alzheimer's disease. PloS one, 6(10):e25446, 2011. 